\title{
Trends in the prevalence of type 2 diabetes and prediabetes in a Japanese community, 1988-2012: the Hisayama Study
}

\author{
Naoko Mukai ${ }^{1,2,3}$. Jun Hata ${ }^{1,2,3} \cdot$ Yoichiro Hirakawa $^{1,2,3} \cdot$ Tomoyuki Ohara $^{2,4}$ - Daigo Yoshida ${ }^{1,2} \cdot$ Udai Nakamura $^{3}$. \\ Takanari Kitazono $^{1,3} \cdot$ Toshiharu Ninomiya ${ }^{1,2}$
}

Received: 12 July 2018 / Accepted: 23 October 2018 / Published online: 3 November 2018

(c) The Japan Diabetes Society 2018

\begin{abstract}
Objective We estimated secular trends in the prevalence of type 2 diabetes (T2DM) and prediabetes, and examined potential explanatory factors for these trends in a Japanese community.

Methods 4 cross-sectional examinations were conducted among subjects aged 40-79 years in $1988(n=2,490), 2002$ $(n=2,856), 2007(n=2,761)$, and $2012(n=2,644)$. Glucose tolerance status was defined by a 75g oral glucose tolerance test. Results The age-standardized prevalence of T2DM increased significantly in both sexes from 1988 to 2002, and thereafter it remained stable in men, and decreased nonsignificantly in women from 2002 to 2012 . The age-standardized prevalence of prediabetes in men increased significantly between 1988 and 2002, but then decreased significantly. A similar trend was observed in women. The age-specific prevalence of T2DM increased greatly in men aged 60-79 years and women aged 70-79 years from 1988 to 2002, and then plateaued at a high level, while a significant decreasing trend was observed in women aged 40-49 years. The mean values of body mass index (BMI) increased steeply in these elderly subjects from 1988 to 2002, and remained at a high level, whereas those in middle-aged women decreased appreciably over the study period.

Conclusions Our findings suggest that in Japanese, there was no further increase in the prevalence of T2DM or prediabetes in either men or women in the 2000s. Secular change in the BMI level was likely to contribute to trends in the prevalence of T2DM, and thus the management of obesity may be important to reduce the prevalence of T2DM.
\end{abstract}

Keywords Type 2 diabetes $\cdot$ Prediabetes $\cdot$ Prevalence $\cdot 75$-g oral glucose tolerance test $\cdot$ Community-based research

Electronic supplementary material The online version of this article (https://doi.org/10.1007/s13340-018-0380-0) contains supplementary material, which is available to authorized users.

Naoko Mukai

n-mukai@cohort.med.kyushu-u.ac.jp

1 Center for Cohort Studies, Graduate School of Medical Sciences, Kyushu University, 3-1-1 Maidashi, Higashi-ku, Fukuoka 812-8582, Japan

2 Department of Epidemiology and Public Health, Graduate School of Medical Sciences, Kyushu University, 3-1-1 Maidashi, Higashi-ku, Fukuoka 812-8582, Japan

3 Department of Medicine and Clinical Science, Graduate School of Medical Sciences, Kyushu University, 3-1-1 Maidashi, Higashi-ku, Fukuoka 812-8582, Japan

4 Department of Neuropsychiatry, Graduate School of Medical Sciences, Kyushu University, 3-1-1 Maidashi, Higashi-ku, Fukuoka 812-8582, Japan

\section{Introduction}

Diabetes is a major public health problem worldwide. The prevalence of diabetes has been growing rapidly, especially in low- and middle-income countries [1]. In regard to highincome Western countries, several epidemiological studies examined trends in the prevalence of diabetes in the 2000s, but their results were inconsistent; the prevalence of diabetes was relatively stable in the United States [2], whereas it continued to increase in the United Kingdom [3], Sweden $[4,5]$, and Canada [6].

A recent global survey reported that Japan, which is one of the high-income Asian countries, has the seventh highest number of individuals with diabetes (about 10.8 million) in the world [1]. However, there have been very few studies investigating trends in the prevalence of diabetes from 2000 or later in Japan. Moreover, there were important limitations in the few available studies. One study [7] diagnosed diabetes based on $\mathrm{HbA}_{1 \mathrm{c}}$ and medical history, which may 
underestimate the true prevalence of diabetes [8]. Another study [9] examined trends in the prevalence of diabetes only until the early 2000s. We have previously reported that the prevalence of type 2 diabetes defined by a 75 -g oral glucose tolerance test (OGTT) increased significantly from 1988 to 2002 in a Japanese community, the town of Hisayama [10]. However, it is unclear whether it continued to increase thereafter. For the present study, we extended the study period from 2002 to 2012 and estimated trends in the prevalence of type 2 diabetes across 4 surveys conducted in 1988, 2002, 2007, and 2012. In addition, some epidemiological studies, including our previous study, have shown that changes in obesity status mainly contribute to secular trends in the prevalence of diabetes [2, 9-11], but other clinical factors, such as heavy alcohol consumption [12], smoking habits [13], and physical inactivity [14], have also been identified as risk factors for developing type 2 diabetes. A better understanding of clinical factors that are potentially responsible for secular change in the prevalence of type 2 diabetes may help to reduce the burden of type 2 diabetes.

The purpose of the present work was to investigate secular trends in the prevalence of type 2 diabetes and prediabetes, which was defined by the OGTT, over a 24 -year period from 1988 to 2012 in a community-dwelling Japanese population. We also examined whether secular change in clinical factors, such as body mass index (BMI), alcohol intake, smoking habits, and regular exercise, was linked with trends in the prevalence of type 2 diabetes.

\section{Methods}

\section{Study population}

A population-based prospective study of cardiovascular disease and its risk factors has been underway since 1961 in the town of Hisayama, a suburb of the Fukuoka metropolitan area on Japan's Kyushu Island [15]. The age and occupational distributions and the nutrient intake of residents in this town have been similar to those of Japan as a whole based on data from the national census and nutrition survey [15]. As a part of the study, 4 cross-sectional diabetes surveys have been conducted on Hisayama residents aged 40-79 years in $1988,2002,2007$, and 2012. In 1988, a total of 2,587 individuals participated in the diabetes survey (participation rate, $80.2 \%$ ). Similarly, the number of participants was 3,028 $(77.7 \%)$ in $2002,2,957(77.1 \%)$ in 2007 , and 2,905 (71.6\%) in 2012. After excluding those who did not consent to participate in the study, those who had already had breakfast, those with missing plasma glucose measurement, and those with a clinical diagnosis of type 1 diabetes, 2,504 subjects in $1988,2,952$ in 2002, 2,899 in 2007, and 2,826 in 2012 were included in the fasting plasma glucose (FPG) sample group
(Supplemental Figure S1). For analyses incorporating 2-h post-load glucose (PG) measurements, additional subjects were excluded if they were not taking oral hypoglycemic agents but were unscreened by OGTT; thus 2,490 subjects in 1988, 2,856 in 2002, 2,761 in 2007, and 2,644 in 2012 were enrolled as an OGTT sample group (Supplemental Figure S1).

\section{Definition of diabetes and prediabetes}

The study subjects underwent the OGTT between 8:00 and 10:30 A.M. after an overnight fast of at least $12 \mathrm{~h}$ in each survey. Plasma glucose concentrations were determined by the glucose-oxidase method in 1988 and 2002, and by the hexokinase method in 2007 and 2012. A previous study reported a high correlation between the plasma glucose values obtained by the glucose-oxidase method and those obtained by the hexokinase method $(r=0.9995)$ [16] $\mathrm{HbA}_{1 \mathrm{c}}$ levels were measured by latex aggregation immunoassay in 2002 and 2007 and by high-performance liquid chromatography in 2012. The values for $\mathrm{HbA}_{1 \mathrm{c}}$ were estimated as National Glycohemoglobin Standardization Program (NGSP) equivalent values and calculated with the formula: $\mathrm{HbA}_{1 \mathrm{c}}(\%)=1.02 \times \mathrm{HbA}_{1 \mathrm{c}}$ (Japan Diabetes Society) (\%) $+0.25 \%$ [17]. Since the $\mathrm{HbA}_{1 \mathrm{c}}$ assay in the 1988 survey was not standardized, we did not use the $\mathrm{HbA}_{1 \mathrm{c}}$ values in the 1988 survey to estimate the prevalence. Glucose tolerance status based on OGTT was defined by the 1998 World Health Organization criteria [18]; namely, for normal glucose tolerance, FPG $<6.1$ and 2-h PG $<7.8$; for impaired fasting glycemia (IFG), FPG 6.1 to 6.9 and 2 -h PG $<7.8$; for impaired glucose tolerance (IGT), FPG $<7.0$ and 2-h PG 7.8-11.0; and for diabetes, FPG $\geq 7.0 \mathrm{mmol} / \mathrm{l}$ or $2-\mathrm{h} \mathrm{PG} \geq 11.1 \mathrm{mmol} / \mathrm{l}$ or both, or the use of antidiabetic medications. Prediabetes was defined as either IFG or IGT. Because there was a variation in the rate of undergoing OGTT across surveys, we also defined diabetes based on FPG levels: FPG $\geq 7.0 \mathrm{mmol} / \mathrm{l}$ or the use of antidiabetic medications. Glucose tolerance status based on $\mathrm{HbA}_{1 \mathrm{c}}$ levels was defined by the American Diabetes Association (ADA) [19] and the National Health and Nutrition Survey (NHNS) of Japan criteria [20]: in the ADA criteria, for normal glucose tolerance, $\mathrm{HbA}_{1 \mathrm{c}}<5.7 \%$; for prediabetes, $\mathrm{HbA}_{1 \mathrm{c}}$ 5.7-6.4\%; and for diabetes, $\mathrm{HbA}_{1 \mathrm{c}} \geq 6.5 \%$ or the use of antidiabetic medications; in the NHNS of Japan criteria, for normal glucose tolerance, $\mathrm{HbA}_{1 \mathrm{c}}<6.0 \%$; for prediabetes, $\mathrm{HbA}_{1 \mathrm{c}}$ 6.0-6.4\%; and for diabetes, $\mathrm{HbA}_{1 \mathrm{c}} \geq 6.5 \%$ or the use of antidiabetic medications.

\section{Clinical evaluation and laboratory measurements}

In the 1988, 2002, 2007, and 2012 surveys, clinical evaluation and laboratory measurements were performed in a similar manner. Serum total and high-density lipoprotein (HDL) 
cholesterols and triglycerides were measured enzymatically in all surveys. In each survey, the height and weight were measured with the subject in light clothes without shoes, and BMI was calculated $\left(\mathrm{kg} / \mathrm{m}^{2}\right)$. Blood pressure was obtained three times using a mercury sphygmomanometer in 1988 and an automated sphygmomanometer in 2002, 2007, and 2012 in a sitting position after rest for at least $5 \mathrm{~min}$; the average values were used in the analyses. Hypertension was defined as a systolic blood pressure $\geq 140 \mathrm{mmHg}$, a diastolic blood pressure $\geq 90 \mathrm{mmHg}$, or current treatment with antihypertensive agents.

Each participant completed a self-administered questionnaire covering medical history, antidiabetic and antihypertensive treatments, alcohol intake, smoking habits, and physical activity. Alcohol intake and smoking habits were classified as either current use or not. Subjects engaging in sports at least three times per week during their leisure time were defined as the regular exercise group.

\section{Statistical analysis}

The SAS software package version 9.4 (SAS Institute, Cary, NC) was used to perform all statistical analyses. The prevalence of type 2 diabetes and each diabetes-related factor was adjusted for the age distribution of the world standard population [21] by using the direct method with 10-year age groupings. The age-adjusted mean values of diabetesrelated factors were calculated using the analysis of covariance method with age taken as a continuous variable. The statistical significance of the difference in the prevalence or mean of each factor between the surveys was assessed using the logistic or linear regression model fit by the generalized estimating equations, respectively, to take into account the individuals contributing to $>1$ examination $[22,23]$. In the above-mentioned analyses, pairwise comparisons versus the 1988 or 2002 survey were adjusted for multiple comparisons by Dunnett test. Serum triglyceride values were transformed into logarithms to improve the skewed distribution. To explore potential explanatory factors for trends in the prevalence of type 2 diabetes, we calculated the age-specific mean values of BMI as well as the age-specific frequencies of alcohol intake, smoking habits, and regular exercise according to 10-year age groups in the four surveys by sex. A value of $p<0.05$ was considered statistically significant in all analyses.

\section{Results}

The age-adjusted mean values or frequencies of diabetesrelated factors in the OGTT sample group among the 4 surveys are shown by sex in Table 1 . The population grew older in both sexes from 1988 to 2012. The mean values of FPG in both sexes and 2-h PG in women increased significantly from 1988 to 2002 and then decreased significantly during the subsequent period from 2002 to 2012, but the mean values of 2-h PG in men increased significantly between 1988 and 2002 and remained on a plateau thereafter. The mean values of $\mathrm{HbA}_{1 \mathrm{c}}$ were significantly elevated from 2002 to 2012 in both sexes. The mean values of HDL cholesterol and the frequencies of antidiabetic medications, alcohol intake, and regular exercise increased significantly from 1988 to 2012 in both sexes. The mean values of BMI in men increased significantly with time, whereas those in women tended to decrease. In men, the mean values of triglycerides and the prevalence of hypertension rose significantly over time, while the frequency of smoking habits decreased significantly. In women, the mean values of total cholesterol decreased significantly between 1988 and 2012, and the frequency of hypertension tended to decrease from 1988 to 2002 and then increased slightly. The mean values of total cholesterol in men and the mean values of triglycerides, and the frequency of smoking habits in women showed no clear secular changes over the study period.

Figure 1 shows the crude and age-standardized prevalence of type 2 diabetes, IGT, and IFG in the OGTT sample group among the 4 surveys. The crude prevalence of type 2 diabetes increased significantly from 1988 to 2002 and showed little change in the subsequent surveys from 2002 to 2012 in both sexes. After controlling for the confounding effects of age, this trend was substantially unchanged in men ( $p<0.001$ from 1988 to 2002, $p$ for trend $=0.35$ from 2002 to 2012), but a nonsignificant decreasing trend was observed in women from 2002 to 2012 ( $p=0.01$ from 1988 to 2002, $p$ for trend $=0.14$ from 2002 to 2012), and the age-standardized prevalence of type 2 diabetes reached $19.4 \%$ for men and $9.1 \%$ for women in 2012. Similar findings were found in the prevalence of type 2 diabetes based on FPG levels in the FPG sample group (Supplemental Figure S2). Meanwhile, the age-standardized prevalence of IFG in both sexes increased from 1988 to 2002 ( $p<0.001$ for men, $p=0.06$ for women), and it decreased significantly from 2002 to 2012 ( $p$ for trend $<0.001$ for men, $p$ for trend $=0.002$ for women). The age-standardized prevalence of IGT in both sexes showed no significant secular change over the study period ( $p$ for trend $=0.84$ for men, $p$ for trend $=0.47$ for women). When IFG and IGT were grouped together as prediabetes, the age-standardized prevalence of prediabetes in men increased significantly from 1988 to $2002(p<0.001)$, and then declined significantly from 2002 to 2012 ( $p$ for trend $<0.001$ ). A similar pattern was observed in the agestandardized prevalence of prediabetes in women, but the differences did not reach a statistically significant level ( $p=0.07$ from 1988 to 2002, $p$ for trend $=0.21$ from 2002 to 2012). Otherwise, the age-standardized prevalence of type 2 diabetes and prediabetes based on $\mathrm{HbA}_{1 \mathrm{c}}$ levels defined 
Table 1 Age-adjusted mean values or frequencies of diabetes-related factors in the OGTT sample group among 4 surveys by sex

\begin{tabular}{|c|c|c|c|c|c|}
\hline Variable & $\begin{array}{l}1988 \\
(n=2,490)\end{array}$ & $\begin{array}{l}2002 \\
(n=2,856)\end{array}$ & $\begin{array}{l}2007 \\
(n=2,761)\end{array}$ & $\begin{array}{l}2012 \\
(n=2,644)\end{array}$ & $P$ for trend \\
\hline \multicolumn{6}{|l|}{ Men } \\
\hline Number of participants & 1,077 & 1,258 & 1,233 & 1,179 & \\
\hline Age, years & $57(10)$ & $59(10)$ & $60(10)^{*}$ & $61(11)^{*}$ & $<0.001$ \\
\hline $\mathrm{FPG}, \mathrm{mmol} / \mathrm{L}$ & $6.0(1.3)$ & $6.3(1.4)^{*}$ & $6.1(1.4)$ & $6.1(1.3)^{*}$ & 0.03 \\
\hline 2-h PG, mmol/L & $7.9(3.9)$ & $8.6(4.2)^{*}$ & $8.5(4.4)^{*}$ & $8.5(4.0)^{*}$ & 0.002 \\
\hline $\mathrm{HbA}_{1 \mathrm{c}}, \%$ & - & $5.5(0.9)$ & $5.6(0.9)^{\dagger}$ & $5.8(0.8)^{\dagger}$ & $<0.001$ \\
\hline Antidiabetic medication, $\%$ & 2.7 & $6.1^{*}$ & $8.3^{*}$ & $9.4^{*}$ & $<0.001$ \\
\hline BMI, $\mathrm{kg} / \mathrm{m}^{2}$ & $22.8(2.9)$ & $23.6(3.0)^{*}$ & $23.6(3.1)^{*}$ & $23.9(3.2)^{*}$ & $<0.001$ \\
\hline Total cholesterol, $\mathrm{mmol} / \mathrm{L}$ & $5.08(1.07)$ & $5.07(0.88)$ & $5.21(0.89)^{*}$ & $5.05(0.89)$ & 0.98 \\
\hline Triglycerides, mmol/L & $1.31(1.27-1.36)$ & $1.34(1.30-1.38)$ & $1.40(1.35-1.45)^{*}$ & $1.38(1.34-1.43)^{*}$ & 0.01 \\
\hline HDL cholesterol, mmol/L & $1.26(0.31)$ & $1.48(0.38)^{*}$ & $1.57(0.42)^{*}$ & $1.52(0.41)^{*}$ & $<0.001$ \\
\hline Hypertension, $\%$ & 42.9 & 42.1 & 44.1 & $45.7 *$ & 0.001 \\
\hline Current drinking, $\%$ & 63.4 & $73.1 *$ & $73.3^{*}$ & $76.2 *$ & $<0.001$ \\
\hline Current smoking, $\%$ & 51.0 & $47.9 *$ & $43.5^{*}$ & $36.2 *$ & $<0.001$ \\
\hline Regular exercise, $\%$ & 11.2 & 11.8 & 12.6 & $15.0 *$ & 0.008 \\
\hline \multicolumn{6}{|l|}{ Women } \\
\hline Number of participants & 1,413 & 1,598 & 1,528 & 1,465 & \\
\hline Age, years & $58(10)$ & $59(11)^{*}$ & $61(10)$ & $61(11)^{*}$ & $<0.001$ \\
\hline $\mathrm{FPG}, \mathrm{mmol} / \mathrm{L}$ & $5.8(1.3)$ & $5.9(1.1)^{*}$ & $5.6(1.1)^{*}$ & $5.7(0.9)$ & $<0.001$ \\
\hline 2-h PG, mmol/L & $7.6(3.3)$ & $7.8(3.5)^{*}$ & $7.5(3.3)$ & $7.5(2.9)$ & 0.04 \\
\hline $\mathrm{HbA}_{1 \mathrm{c}}, \%$ & - & $5.4(0.8)$ & $5.5(0.7)^{\dagger}$ & $5.7(0.6)^{\dagger}$ & $<0.001$ \\
\hline Antidiabetic medication, $\%$ & 2.3 & 2.8 & $4.4^{*}$ & $5.0^{*}$ & $<0.001$ \\
\hline BMI, $\mathrm{kg} / \mathrm{m}^{2}$ & $23.1(3.2)$ & $23.1(3.5)$ & $22.9(3.6)$ & $22.8(3.7)$ & 0.06 \\
\hline Total cholesterol, $\mathrm{mmol} / \mathrm{L}$ & $5.57(1.05)$ & $5.46(0.90)^{*}$ & $5.64(0.92)$ & $5.45(0.94)^{*}$ & 0.007 \\
\hline Triglycerides, mmol/L & $1.08(1.06-1.11)$ & $1.03(1.01-1.05)^{*}$ & $1.07(1.04-1.09)^{*}$ & $1.04(1.01-1.06)^{*}$ & 0.08 \\
\hline HDL cholesterol, mmol/L & $1.33(0.29)$ & $1.73(0.41)^{*}$ & $1.87(0.46)^{*}$ & $1.80(0.44)^{*}$ & $<0.001$ \\
\hline Hypertension, \% & 32.7 & 30.1 & 32.7 & 31.4 & 0.007 \\
\hline Current drinking, $\%$ & 9.7 & $30.4^{*}$ & $40.3^{*}$ & $41.7^{*}$ & $<0.001$ \\
\hline Current smoking, $\%$ & 6.7 & $8.4^{*}$ & $10.3 *$ & 9.3 & 0.37 \\
\hline Regular exercise, $\%$ & 8.6 & 8.6 & 9.9 & $11.0 *$ & $<0.001$ \\
\hline
\end{tabular}

Age is not age-adjusted. Triglycerides are shown by geometric means and 95\% confidence intervals due to the skewed distribution. All other values are given as the mean (standard deviations) or as a percentage

$O G T T$ oral glucose tolerance test, $F P G$ fasting plasma glucose, $P G$ post-load glucose, $B M I$ body mass index, $H D L$ high-density lipoprotein

$* p<0.05$ compared with the examination in 1988

${ }^{\dagger} p<0.05$ compared with the examination in 2002

by both ADA and NHNS of Japan criteria increased significantly from 2002 to 2012 in both sexes (type 2 diabetes: $p$ for trend $<0.001$ for men, $p$ for trend $=0.03$ for women; prediabetes: both $\mathrm{p}$ for trend $<0.001$ ) (Supplemental Figures $\mathrm{S} 3$ and $\mathrm{S} 4$ ).

Figure 2 demonstrates the prevalence of type 2 diabetes according to 10 -year age groups in the 4 surveys. The prevalence of type 2 diabetes increased significantly from 1988 to 2002 in men aged 60-69 and 70-79 years (both $p<0.001)$, and in women aged 70-79 years $(p=0.01)$, and remained similar during the subsequent period from 2002 to 2012. In contrast, the prevalence of type 2 diabetes in women aged 40-49 years showed a decreasing trend from 2002 to 2012 ( $p$ for trend $=0.03$ ) and the difference between 2002 and 2012 was significant $(p=0.02)$. There was no clear secular change in the prevalence of type 2 diabetes in men aged 40-59 years and women aged 50-69 years. The prevalence of prediabetes defined by OGTT increased from 1988 to 2002 in men aged 40-49 $(p=0.06)$ and $50-59$ years $(p<0.001)$, and then declined significantly from 2002 to 2012 (both $p$ for trend $<0.001$ ) (Supplemental Figure S5). The prevalence of prediabetes in women aged 40-49 years continued to decrease from 1988 to 2012 ( $p$ for trend $=0.002$ ). 
a Crude prevalence

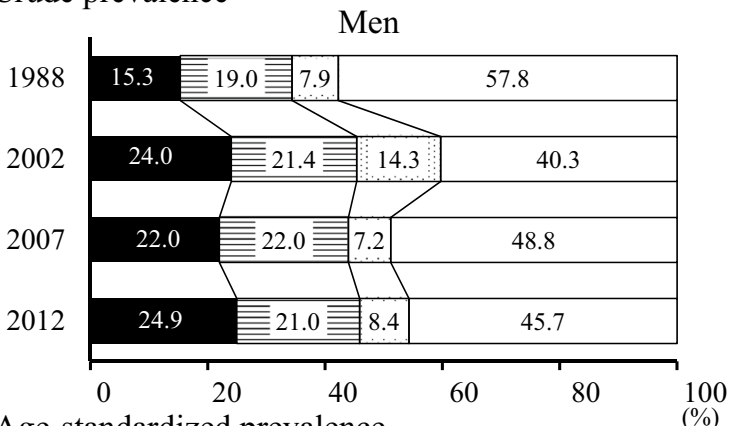

b Age-standardized prevalence

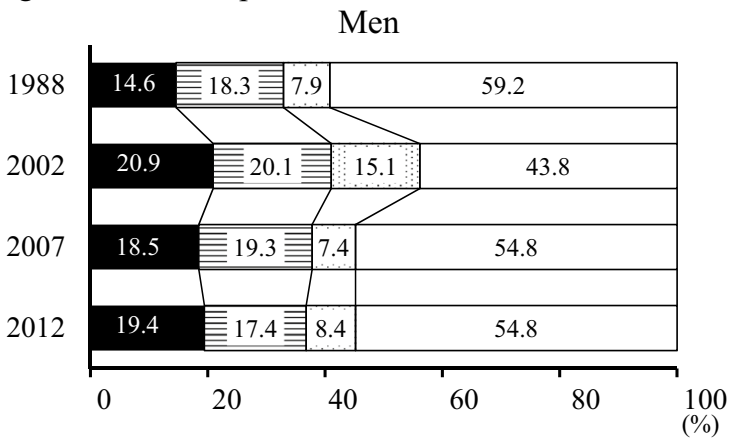

Type 2 diabetes $\quad$ Impaired glucose tolerance

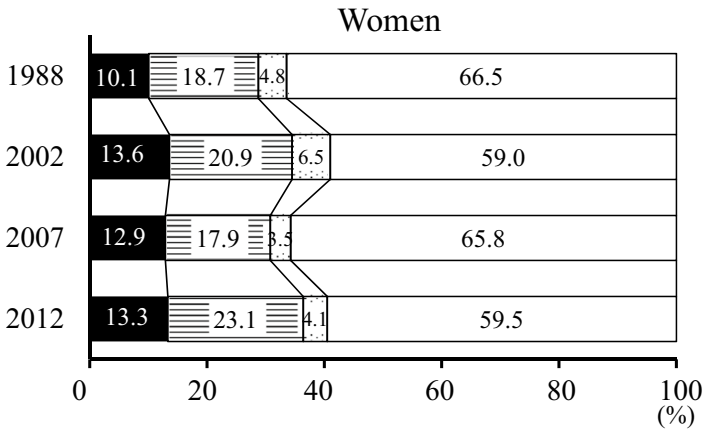

Women

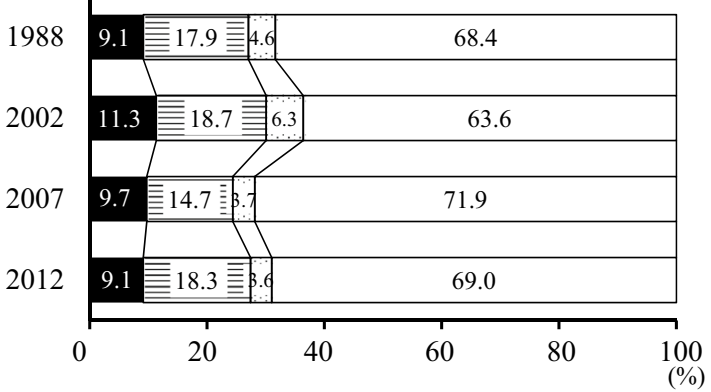

Impaired fasting glycemia $\square$ Normal glucose tolerance

Fig. 1 Secular trends in the crude (a) and age-standardized (b) prevalence of type 2 diabetes, impaired glucose tolerance, and impaired fasting glycemia in the OGTT sample group in 1988, 2002, 2007, and 2012 by sex

Fig. 2 Secular trends in the prevalence of type 2 diabetes according to age groups in the OGTT sample group in 1988, 2002,2007 , and 2012 by sex. ${ }^{*} p<0.05$ versus $1988,{ }^{\#} p<0.05$ versus $2002,{ }^{\dagger} p$ for trend $<0.01$ from 1988 to 2012 , ${ }^{\ddagger} \mathrm{p}$ for trend $<0.05$ from 2002 to 2012
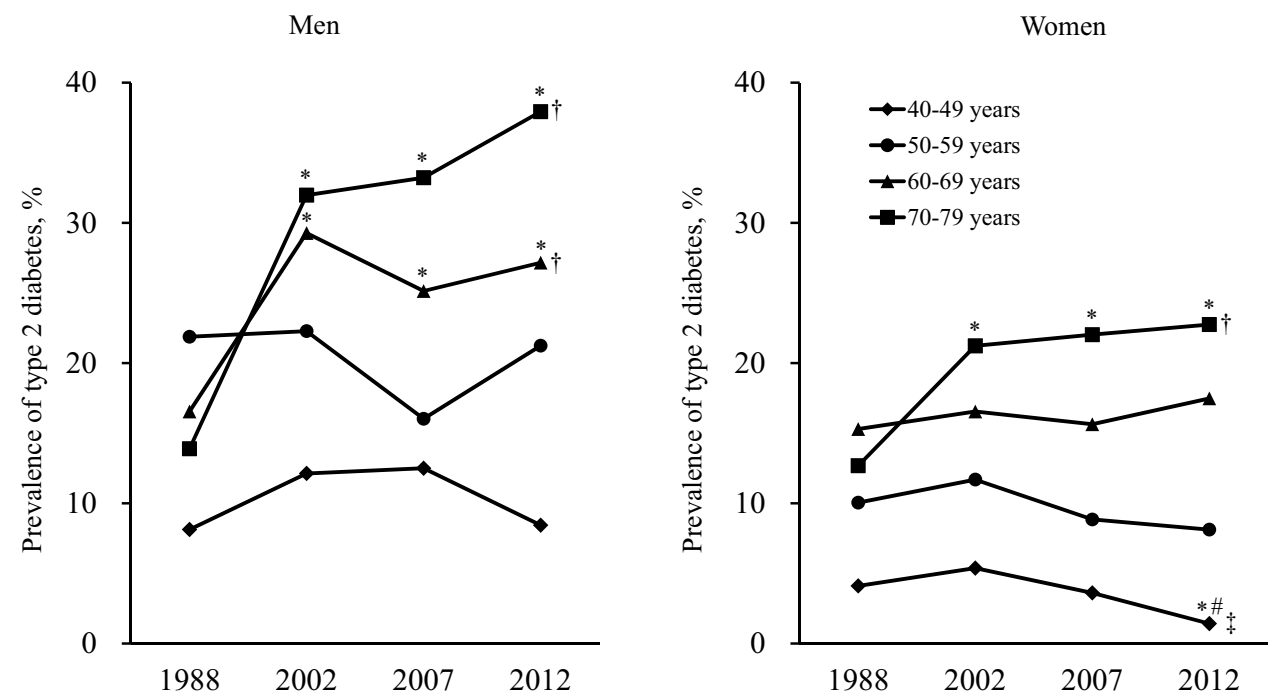

Finally, we compared the age-specific mean values of BMI (Fig. 3) and the age-specific frequencies of alcohol intake, smoking habits, and regular exercise (Supplemental Figs S6-S8) among the four surveys by sex. For men, the mean values of BMI increased consistently in the two oldest age ranges (60-69 and 70-79 years) over the period from 1988 to 2012 (both $p$ for trend $<0.001$ ). Meanwhile, the mean values of BMI in middle-aged men (40-49 and
50-59 years) also increased significantly from 1988 to 2002 (both $p<0.05$ ), but this increasing trend slowed in the subsequent period. For women, the mean values of BMI increased steeply in the age bracket of 70-79 years from 1988 to $2002(p<0.001)$, and these elderly subjects continued to maintain a higher BMI level. In contrast, a decreasing trend was observed in women aged 40-49 and 50-59 years over the study period and the mean values of BMI in these 
Fig. 3 Secular trends in the mean values of body mass index according to age groups in the OGTT sample group in 1988, 2002, 2007, and 2012 by sex. $* p<0.05$ versus 1988 , $\# p<0.05$ versus $2002,{ }^{\dagger} \mathrm{p}$ for trend $<0.05$ from 1988 to 2012 , ${ }^{\ddagger}$ p for trend $<0.05$ from 2002 to 2012

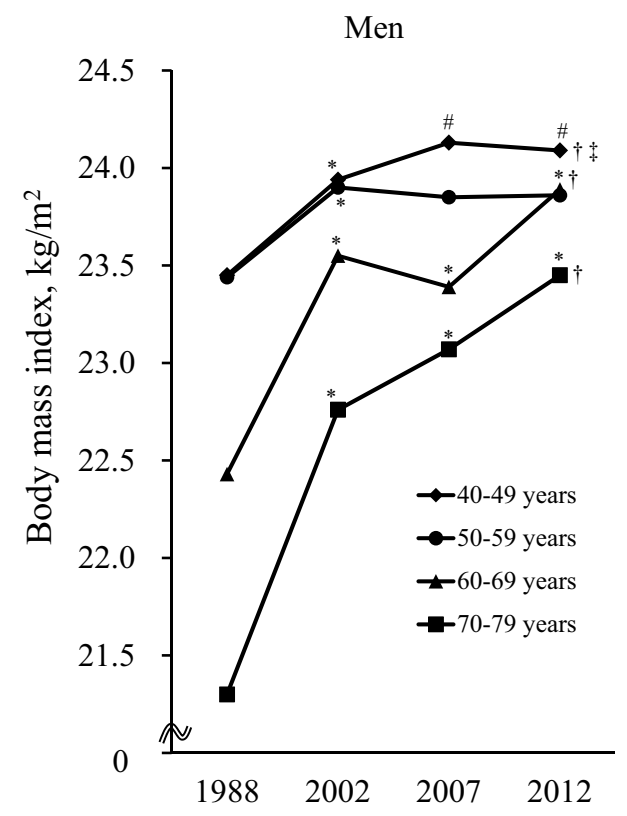

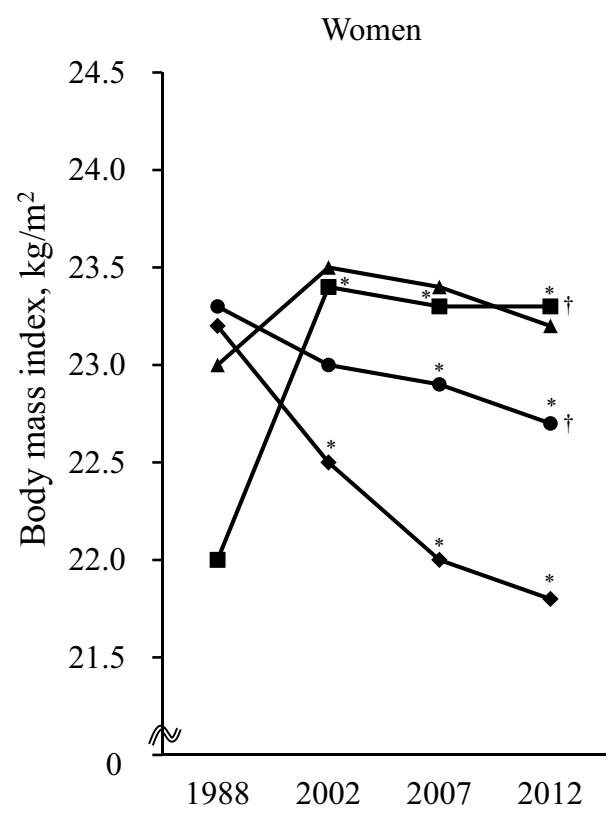

middle-aged women in 2012 were significantly lower than in those in 1988 (both $p<0.01$ ). Meanwhile, trends in the age-and sex-specific frequencies of alcohol intake, smoking habits, and regular exercise were not linked with the patterns of type 2 diabetes.

\section{Discussion}

To our knowledge, this 24-year repeated cross-sectional analysis is the first study to examine secular trends in the prevalence of type 2 diabetes and prediabetes defined by the OGTT over a long period of time in a Japanese community. Among the 4 cross-sectional surveys, the age-standardized prevalence of type 2 diabetes increased significantly in both sexes from 1988 to 2002, and thereafter it remained relatively stable in men, but tended to decrease in women from 2002 to 2012. The age-standardized prevalence of prediabetes in both sexes showed an increasing trend between 1988 and 2002, and then a decreasing trend between 2002 and 2012. In the age-stratified analysis, the prevalence of type 2 diabetes in the elderly increased greatly from 1988 to 2002 , and then plateaued at a high level. In contrast, the prevalence of type 2 diabetes in middle-aged women declined from 2002 through 2012.

The present study showed that the prevalence of type 2 diabetes did not change in men and tended to decrease in women from 2002 to 2012, and that the prevalence of prediabetes decreased in both sexes during the same period. The NHNS of Japan reported that the prevalence of diabetes remained on a plateau between 2003 and 2012 [7] and that there was little change in the prevalence of prediabetes in men and a decrease in that in women between 2007 and 2012 [20]. These findings suggest that there was no upward trend in the prevalence of diabetes and prediabetes from the 2000s through the 2010s in the Japanese population. Other Asian population studies showed an increasing trend in the prevalence of diabetes between 2007-2008 and 2013 in a Chinese population [24, 25] and between 1999-2002 and 2008-2015 in Asian Indians [26, 27], while a national study in South Korea reported that the prevalence of diabetes did not change in men, but decreased in younger women between 2001 and 2010 [11], which is consistent with our findings. Taken together, these findings indicate that the prevalence of diabetes in the 2000s plateaued or declined in high-income Asian countries, whereas an increasing trend persisted in middle-income Asian countries.

Our age-stratified analysis indicated that the prevalence of type 2 diabetes decreased with time in middle-aged women after 2002, whereas it increased steeply in elderly participants in both sexes from 1988 to 2002, and then plateaued at a high level. In addition, the prevalence of prediabetes decreased mainly in the middle-age group for both sexes from 2002 to 2012. These trends in type 2 diabetes and prediabetes showed similar patterns of BMI levels according to age groups, i.e., an elevated BMI level in the elderly, a slowdown in the BMI increase in middle-aged men, and a large reduction of the BMI level among middle-aged women in recent years. Because obesity is well-established as a strong risk factor for type 2 diabetes, secular change in the BMI level was likely to contribute to secular trends in the prevalence of type 2 diabetes and prediabetes. Thus, our findings suggest that management of obesity may reduce the prevalence of type 2 diabetes in the Japanese population. 
The prevalence of type 2 diabetes and prediabetes in our 2012 survey (men $19.4 \%$; women $9.1 \%$ for type 2 diabetes; men $25.8 \%$; women $21.9 \%$ for prediabetes) was higher than those estimated in the NHNS of Japan for the same year (men $15.2 \%$; women $8.7 \%$ for diabetes; men $12.1 \%$; women $13.1 \%$ for prediabetes) [20]. This diversity may have arisen from a difference in the definition for diabetes between the 2 studies; the OGTT for determining glucose tolerance status was used in the present study, whereas the latter study used a measurement of $\mathrm{HbA}_{1 \mathrm{c}}$ and medical history, not of plasma glucose. Recent large pooled analyses have revealed that using $\mathrm{HbA}_{1 \mathrm{c}}$ measurement alone missed a substantial proportion of the subjects with undiagnosed diabetes defined by OGTT [8]. Indeed, our sensitivity analysis using $\mathrm{HbA}_{1 \mathrm{c}}$ based on the NHNS of Japan criteria revealed that the agestandardized prevalences of type 2 diabetes and prediabetes were $13.0 \%$ and $7.9 \%$ for men and $6.6 \%$ and $8.3 \%$ for women, respectively, in the 2012 survey, which were lower than the prevalences obtained using the OGTT-based definition, and also lower than those in the NHNS of Japan in 2012. Moreover, in the present study, the prevalence of type 2 diabetes and prediabetes diagnosed by $\mathrm{HbA}_{1 \mathrm{c}}$ levels increased significantly from 2002 to 2012, which is inconsistent with the results obtained using OGTT. A previous epidemiological study [28] reported poor agreement between $\mathrm{HbA}_{1 \mathrm{c}}$ and OGTT in classifying glucose tolerance status. In addition, a study of nondiabetic subjects [29] showed that $\mathrm{HbA}_{1 \mathrm{c}}$ levels increased with age even after multivariate adjustments for FPG and 2-h PG. Because our study population grew older with time, the glucose-independent effects of aging on $\mathrm{HbA}_{1 \mathrm{c}}$ levels would contribute to an increasing prevalence estimated by $\mathrm{HbA}_{1 \mathrm{c}}$ levels. These might be reasons for the discrepant findings between the $\mathrm{HbA}_{1 \mathrm{c}}$ - and OGTT-based definitions.

The strengths of our study include the long observational period, the high participation rate and the use of an OGTT for determining glucose tolerance status in each survey. However, some limitations should be mentioned. First, the diagnosis of glucose tolerance status was based on a single measurement of glucose levels, as was the case in most other epidemiological studies. This limitation may have caused misclassification of glucose tolerance categories. However, we believe that such misclassification would have occurred at a similar level across the surveys. Second, there may have been a selection bias due to the exclusion of subjects who did not undergo the OGTT. However, similar trends in the prevalence of type 2 diabetes were found using FPG levels as the basis in the FPG sample group. Third, the participation rate of the health examination in 2012 was lower than that in the other 3 examinations, and this might have increased the risk of selection bias in the 2012 survey. It is generally acknowledged that an acceptable participation rate in a population-based study, i.e., a rate that practically eliminates the risk of selection bias attributable to non-participants, is above $70 \%$ of the target population $[30,31]$. We enrolled more than $70 \%$ of residents in every examination, and, therefore, we believe that our findings reflect actual trends in the prevalence in our population.

In conclusion, the present study suggests that in a Japanese population, there was no further increase in the prevalence of type 2 diabetes or prediabetes in either men or women in the 2000s. Meanwhile, the prevalence of type 2 diabetes was still high in the elderly subjects, but decreased significantly in middle-aged women. These trends could be explained by the change in the BMI level. Our findings indicate that the management of obesity may be important to reduce the prevalence of type 2 diabetes in Japanese.

Acknowledgements The authors thank the staff of the Division of Health and Welfare of Hisayama for their cooperation in this study. This study was supported in part by Grants-in-Aid for Scientific Research (A) (JP16H02644, and JP16H02692),(B) (JP16H05850, JP16H05557, JP17H04126, and JP18H02737) and (C) (JP16K09244, JP17K09114, JP17K09113, JP17K01853, JP18K07565, and JP18K09412) and (Early-Career Scientists) (JP18K17925, and JP18K17382) from the Ministry of Education, Culture, Sports, Science and Technology of Japan; by Health and Labour Sciences Research Grants of the Ministry of Health, Labour and Welfare of Japan (H29-Junkankitou-Ippan-003, and H30-Shokuhin-[Sitei]-005); and by the Japan Agency for Medical Research and Development (JP18dk0207025, JP18ek0210082, JP18gm0610007, JP18ek0210083, JP18km0405202, JP18ek0210080, JP18fk0108075).

\section{Compliance with ethical standards}

Conflict of interest The authors declare that there is no conflict of interest associated with this manuscript.

Human rights statement All procedures followed were in accordance with the ethical standards of the responsible committee on human experimentation (the Kyushu University Institutional Review Board for Clinical Research, date of approval: 28 July 2017, approval no. 29-213) and with the Helsinki Declaration of 1964 and later versions.

Informed consent Informed consent was obtained from the participants before they were included in the study.

\section{References}

1. Collaboration NRF. Worldwide trends in diabetes since 1980: a pooled analysis of 751 population-based studies with 44 million participants. Lancet. 2016;387:1513-30.

2. Menke A, Casagrande S, Geiss L, Cowie CC. Prevalence of and trends in diabetes among adults in the United States, 1988-2012. JAMA. 2015;314:1021-9.

3. Zghebi SS, Steinke DT, Carr MJ, Rutter MK, Emsley RA, Ashcroft DM. Examining trends in type 2 diabetes incidence, prevalence and mortality in the UK between 2004 and 2014. Diabetes Obes Metab. 2017;19:1537-45.

4. Jansson SP, Fall K, Brus O, Magnuson A, Wandell P, Ostgren CJ, Rolandsson O. Prevalence and incidence of diabetes mellitus: a 
nationwide population-based pharmaco-epidemiological study in Sweden. Diabet Med. 2015;32:1319-28.

5. Carlsson S, Andersson T, Ahlbom A. Prevalence and incidence of diabetes mellitus: a nationwide population-based pharmacoepidemiological study in Sweden. Diabet Med. 2016;33:1149-50.

6. Lipscombe LL, Hux JE. Trends in diabetes prevalence, incidence, and mortality in Ontario, Canada 1995-2005: a population-based study. Lancet. 2007;369:750-6.

7. Ikeda N, Nishi N, Noda H, Noda M. Trends in prevalence and management of diabetes and related vascular risks in Japanese adults: Japan National Health and Nutrition Surveys 2003-2012. Diabetes Res Clin Pract. 2017;127:115-22.

8. Collaboration NRF. Effects of diabetes definition on global surveillance of diabetes prevalence and diagnosis: a pooled analysis of 96 population-based studies with 331,288 participants. Lancet Diabetes Endocrinol. 2015;3:624-37.

9. Kabeya Y, Kato M, Isogawa A, Takahashi Y, Matsushita Y, Goto A, Iso $\mathrm{H}$, Inoue $\mathrm{M}$, Mizoue $\mathrm{T}$, Tsugane $\mathrm{S}$, Kadowaki T, Noda M. Descriptive epidemiology of diabetes prevalence and $\mathrm{HbA} 1 \mathrm{c}$ distributions based on a self-reported questionnaire and a health checkup in the JPHC diabetes study. J Epidemiol. 2014;24:460-8.

10. Mukai N, Doi Y, Ninomiya T, Hirakawa Y, Nagata M, Yoshida D, Hata J, Fukuhara M, Nakamura U, Kitazono T, Kiyohara Y. Trends in the prevalence of type 2 diabetes and prediabetes in community-dwelling Japanese subjects: the Hisayama Study. J Diabetes Investig. 2014;5:162-9.

11. Koo BK, Kim EK, Choi H, Park KS, Moon MK. Decreasing trends of the prevalence of diabetes and obesity in Korean women aged 30-59 years over the past decade: results from the Korean National Health and Nutrition Examination Survey, 2001-2010. Diabetes Care. 2013;36:e95-6.

12. Baliunas DO, Taylor BJ, Irving H, Roerecke M, Patra J, Mohapatra S, Rehm J. Alcohol as a risk factor for type 2 diabetes: a systematic review and meta-analysis. Diabetes Care. 2009;32:2123-32.

13. Willi C, Bodenmann P, Ghali WA, Faris PD, Cornuz J. Active smoking and the risk of type 2 diabetes: a systematic review and meta-analysis. JAMA. 2007;298:2654-64.

14. Wilmot EG, Edwardson CL, Achana FA, Davies MJ, Gorely T, Gray LJ, Khunti K, Yates T, Biddle SJ. Sedentary time in adults and the association with diabetes, cardiovascular disease and death: systematic review and meta-analysis. Diabetologia. 2012;55:2895-905.

15. Hata J, Ninomiya T, Hirakawa $Y$, Nagata M, Mukai N, Gotoh S, Fukuhara M, Ikeda F, Shikata K, Yoshida D, Yonemoto K, Kamouchi M, Kitazono T, Kiyohara Y. Secular trends in cardiovascular disease and its risk factors in Japanese: half century data from the Hisayama Study, 1961-2009. Circulation. 2013;128:1198-205.

16. Neeley WE. Simple automated determination of serum or plasma glucose by a hexokinase/glucose-6-phosphate dehydrogenase method. Clin Chem. 1972;18:509-15.

17. Committee on the standardization of diabetes mellitus-related laboratory testing of Japan Diabetes Society. International clinical harmonization of glycated hemoglobin in Japan: from Japan Diabetes Society to National Glycohemoglobin Standardization Program values. J Diabetes Investig. 2012;3:39-40.

18. Alberti KG, Zimmet PZ. Definition, diagnosis and classification of diabetes mellitus and its complications. Part 1: diagnosis and classification of diabetes mellitus provisional report of a WHO consultation. Diabet Med. 1998;15:539-53.

19. American Diabetes Association. Diagnosis and classification of diabetes mellitus. Diabetes Care. 2010;33(Suppl 1):S62-9.

20. Ministry of Health, Labour and Welfare. The National Health and Nutrition Survey in Japan, 2012. 2014 (in Japanese).

21. Ahmad OB, Boschi-Pinto C, Lopez AD, Murray CJ, Lozano R, Inoue M. Age standardization of rates: a new WHO standard. Geneva: World Health Organization; 2001.

22. Liang KY, Zeger SL. Longitudinal data analysis using generalized linear models. Biometrics. 1986;78:13-22.

23. Wolf PA, Benjamin EJ, Belanger AJ, Kannel WB, Levy D, D'Agostino RB. Secular trends in the prevalence of atrial fibrillation: the Framingham Study. Am Heart J. 1996;131:790-5.

24. Yang W, Lu J, Weng J, Jia W, Ji L, Xiao J, Shan Z, Liu J, Tian H, Ji Q, Zhu D, Ge J, Lin L, Chen L, Guo X, Zhao Z, Li Q, Zhou Z, Shan G, He J. Prevalence of diabetes among men and women in China. N Engl J Med. 2010;362:1090-101.

25. Wang L, Gao P, Zhang M, Huang Z, Zhang D, Deng Q, Li Y, Zhao Z, Qin X, Jin D, Zhou M, Tang X, Hu Y, Wang L. Prevalence and ethnic pattern of diabetes and prediabetes in China in 2013. JAMA. 2017;317:2515-23.

26. Sadikot SM, Nigam A, Das S, Bajaj S, Zargar AH, Prasannakumar KM, Sosale A, Munichoodappa C, Seshiah V, Singh SK, Jamal A, Sai K, Sadasivrao Y, Murthy SS, Hazra DK, Jain S, Mukherjee S, Bandyopadhay S, Sinha NK, Mishra R, Dora M, Jena B, Patra P, Goenka K. The burden of diabetes and impaired glucose tolerance in India using the WHO 1999 criteria: prevalence of diabetes in India study (PODIS). Diabetes Res Clin Pract. 2004;66:301-7.

27. ICMR-INDIAB Collaborative Study Group. Prevalence of diabetes and prediabetes in 15 states of India: results from the ICMRINDIAB population-based cross-sectional study. Lancet Diabetes Endocrinol. 2017;5:585-96.

28. Olson DE, Rhee MK, Herrick K, Ziemer DC, Twombly JG, Phillips LS. Screening for diabetes and pre-diabetes with proposed A1C-based diagnostic criteria. Diabetes Care. 2010;33:2184-9.

29. Pani LN, Korenda L, Meigs JB, Driver C, Chamany S, Fox CS, Sullivan L, D'Agostino RB, Nathan DM. Effect of aging on A1C levels in individuals without diabetes: evidence from the Framingham Offspring Study and the National Health and Nutrition Examination Survey 2001-2004. Diabetes Care. 2008;31:1991-6.

30. Groves RM. Survey errors and survey costs. New York: Wiley; 1989.

31. Kasper JD, Shapiro S, Guralnik JM, Bandeen-Roche KJ, Fried LP. Designing a community study of moderately to severely disabled older women: the Women's Health and Aging Study. Ann Epidemiol. 1999;9:498-507. 